\title{
Adaptive Fuzzy Sliding Mode Control of MEMS Gyroscope with Finite Time Convergence
}

\author{
Jianxin Ren, Rui Zhang, and Bin Xu \\ School of Automation, Northwestern Polytechnical University, Xi'an 710129, China \\ Correspondence should be addressed to Bin Xu; smileface.binxu@gmail.com
}

Received 25 April 2016; Accepted 18 July 2016

Academic Editor: José A. Somolinos

Copyright (C) 2016 Jianxin Ren et al. This is an open access article distributed under the Creative Commons Attribution License, which permits unrestricted use, distribution, and reproduction in any medium, provided the original work is properly cited.

\begin{abstract}
This paper presents adaptive fuzzy finite time sliding mode control of microelectromechanical system gyroscope with uncertainty and external disturbance. Firstly, fuzzy system is employed to approximate the uncertainty nonlinear dynamics. Secondly, nonlinear sliding mode hypersurface and double exponential reaching law are selected to design the finite time convergent sliding mode controller. Thirdly, based on Lyapunov methods, adaptive laws are presented to adjust the fuzzy weights and the system can be guaranteed to be stable. Finally, the effectiveness of the proposed method is verified with simulation.
\end{abstract}

\section{Introduction}

MEMS gyroscopes have become the most growing microsensors in recent years due to the characteristics of compact size, low cost, and high sensitivity. Most MEMS gyroscopes sales in the market are vibrating silicon micromechanical gyroscopes, whose basic principle is to generate and detect Coriolis Effect. As depicted in Figure 1, under assumption that the proof mass $m$ of gyroscope rotates around $z$-axis at a speed of $\vec{\Omega}$ and makes uniform motion along the $x$-axis at a speed of $\vec{\nu}$, a Coriolis force of $\vec{F}=-2 m \vec{\Omega} \times \vec{\nu}$ is produced along $y$-axis.

In the last few years, numerous advanced control approaches with intelligent design have been studied to realize the trajectory tracking in [1-4] and to handle the system parametric uncertainties and disturbances, and the adaptive control can be found in [5-7]. For control of MEMS gyroscope, Park and Horowitz firstly applied adaptive state feedback control method [8]. Both drive shaft and sensitive axis were subjected to feedback control force in this control method, which administered two axial modal vibration track specified reference trajectories, weakening the boundary between drive mode and test mode as well.

Sliding mode control changes its structure to force the system in accordance with a predetermined trajectory. Batur et al. developed a sliding mode control for MEMS gyroscope system in [9]. Since then, adaptive sliding mode control approach with the advantages of variable structure methods and adaptive control strategies are presented to control MEMS gyroscopes in [10, 11].

Due to the necessity of ideal sliding mode, good dynamic quality, and high robustness, several methods are extended to improve the performance. $\mathrm{Yu}$ and Man investigated a nonlinear sliding mode hypersurface to ensure that systems from any point of the sliding mode surface were able to reach the balance point in a limited time in [12-16]. Bartoszewicz [17] examined the reaching laws introduced by Gao and Hung in [18] and proposed an enhanced version of those reaching laws, which was more appropriate for systems subject to constraints. Recently, Fallaha et al. studied a novel approach, which allowed chattering reduction on control input while keeping tracking performance in steady-state regime [19]. This approach consisted of designing a nonlinear reaching law by using an exponential function that dynamically adapted to the variations of the controlled system. Mei and Wang in [20] proposed a nonlinear sliding mode surface which converged to the equilibrium point with a higher speed than both linear sliding mode surface and terminal sliding mode surface. In addition, a new two-power reaching law was proposed to make the system move toward the sliding mode faster.

As a matter of fact, the methods mentioned above are highly dependent on the structure of the nonlinearity, while, 


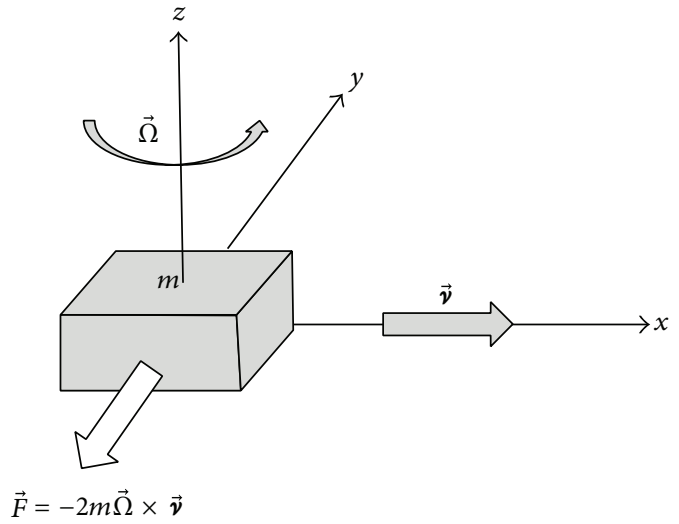

FIGURE 1: Coriolis Effect.

currently, accurate model is unavailable. Thus, fuzzy model has been widely used to approximate nonlinear objects in $[21,22]$. Robust adaptive sliding mode control with on-line identification for the upper bounds of external disturbance and estimator for the nonlinear dynamics of MEMS gyroscope uncertainty parameters was proposed in [23].

In this paper, an adaptive fuzzy sliding mode control strategy with nonlinear sliding mode hypersurface and double exponential reaching law is developed to track MEMS gyroscope. Furthermore, it converges faster compared with strategies using conventional sliding mode surface in [23] and terminal sliding mode surface in [12-16].

The rest of this paper is organized as follows. The dynamics of MEMS gyroscope with parametric uncertainties and disturbances are given in Section 2. Controller design and stability analysis are discussed in Section 3. Numerical simulations are conducted to verify the superiority of the proposed approach in Section 4, compared with conventional adaptive fuzzy sliding mode control. Conclusions are drawn in Section 5.

\section{Dynamics of MEMS Gyroscope}

The basic principle of $z$-axis vibratory MEMS gyroscope is shown in Figure 2, which can be described as a qualitystiffness-damping system. Owing to mechanical coupling caused by fabrication imperfections, the dynamics can be derived as

$$
\begin{aligned}
& m \ddot{x}+d_{x x} \dot{x}+\left(d_{x y}-2 m \Omega_{z}^{*}\right) \dot{y}+\left(k_{x x}-m \Omega_{z}^{*^{2}}\right) x \\
& +k_{x y} y=u_{x}^{*}, \\
& m \ddot{y}+d_{x x} \dot{y}+\left(d_{x y}+2 m \Omega_{z}^{*}\right) \dot{x}+\left(k_{y y}-m \Omega_{z}^{*^{2}}\right) y \\
& +k_{x y} x=u_{y}^{*},
\end{aligned}
$$

where $m$ is the mass of proof mass; $\Omega_{z}^{*}$ is the input angular velocity; $x, y$ represent the system generalized coordinates; $d_{x x}, d_{y y}$ represent damping terms; $d_{x y}$ represents asymmetric damping term; $k_{x x}, k_{y y}$ represent spring terms; $k_{x y}$ represents

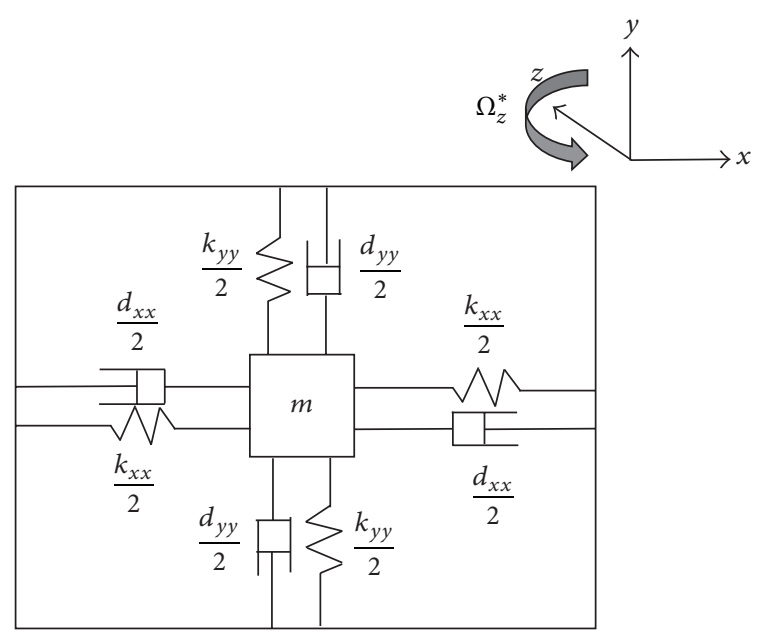

FIGURE 2: The basic principle diagram of $z$-axis vibratory MEMS gyroscope.

asymmetric spring terms; and $u_{x}^{*}, u_{y}^{*}$ represent the control forces.

On issues related to the study of mechanism, the law described by model is required to be independent of dimensions. So, it is necessary to establish nondimensional vector dynamics. Because of the nondimensional time $t^{*}=\omega_{o} t$, both sides of (1) should be divided by reference frequency $\omega_{o}^{2}$, reference length $q_{o}$, and reference mass $m$. Then the dynamics can be rewritten in vector forms:

$$
\begin{aligned}
\frac{q^{*}}{q_{o}} & +\frac{D^{*}}{m \omega_{o}} \frac{q^{*}}{q_{o}}+2 \frac{S^{*}}{\omega_{o}} \frac{q^{*}}{q_{o}}-\frac{\Omega_{z}^{*^{2}}}{\omega_{o}^{2}} \frac{q^{*}}{q_{o}}+\frac{K_{1}^{*}}{m \omega_{o}^{2}} \frac{q^{*}}{q_{o}} \\
& =\frac{u^{*}}{m \omega_{o}^{2} q_{o}},
\end{aligned}
$$

where $q^{*}=\left[\begin{array}{l}x \\ y\end{array}\right], u^{*}=\left[\begin{array}{l}u_{x}^{*} \\ u_{y}^{*}\end{array}\right], D^{*}=\left[\begin{array}{ll}d_{x x} & d_{x y} \\ d_{x y} & d_{y y}\end{array}\right], S^{*}=\left[\begin{array}{cc}0 & -\Omega_{z}^{*} \\ \Omega_{z}^{*} & 0\end{array}\right]$, $K_{1}^{*}=\left[\begin{array}{ll}k_{x x} & k_{x y} \\ k_{x y} & k_{y y}\end{array}\right]$.

New parameters are defined as follows:

$$
\begin{aligned}
q & =\frac{q^{*}}{q_{o}}, \\
u & =\frac{u^{*}}{m \omega_{o}^{2} q_{o}}, \\
\Omega_{z} & =\frac{\Omega_{z}^{*}}{\omega_{o}}, \\
D & =\frac{D^{*}}{m \omega_{o}}, \\
K_{1} & =\frac{K_{1}^{*}}{m \omega_{o}^{2}}, \\
S & =-\frac{S^{*}}{\omega_{o}} .
\end{aligned}
$$


Thus, the final form of the nondimensional vector dynamics is

$$
\ddot{q}=(2 S-D) \dot{q}+\left(\Omega_{z}^{2}-K_{1}\right) q+u \text {. }
$$

In presence of parametric uncertainties and external disturbance, based on (4), state equation of dynamics is established as

$$
\ddot{q}=(A+\Delta A) \dot{q}+(B+\Delta B) q+C u+d(t),
$$

where $A \in R^{2 \times 2}, B \in R^{2 \times 2}, C \in R^{2 \times 2}$ are system known matrices; $\Delta A, \Delta B$ are parametric uncertainties; and $d(t)$ is an external disturbance. Besides, $A=2 S-D, B=\Omega_{z}^{2}-K_{1}$.

If the system total interference (consisting of parametric uncertainties and external disturbance) is represented by $P(t)$, we know

$$
\ddot{q}=A \dot{q}+B q+C u+P(\dot{q}, q, t),
$$

where $P(\dot{q}, q, t)=\Delta A \dot{q}+\Delta B q+d(t)$.

It is vital that (6) must meet the following assumptions.

Assumption 1. The total interference $\|P(\dot{q}, q, t)\| \leq P_{c}$, where $P_{c}$ is an unknown positive vector.

Assumption 2. The total interference $P(\dot{q}, q, t)$ meets sliding mode matching conditions; namely, $\Delta A=\mathrm{CH}_{1}, \Delta B=\mathrm{CH}_{2}$, $d(t)=\mathrm{CH}_{3}$, where $\mathrm{H}_{1}, \mathrm{H}_{2}, \mathrm{H}_{3}$ are unknown matrices with appropriate dimensions.

Assumption 3. A, B are observability matrices.

Based on the above assumptions, the controller can be designed to compensate the total interference.

\section{Adaptive Fuzzy Finite Time Sliding Mode Control}

The fuzzy model of $P(t)$ could be composed of $M$ IF-THEN rules, and the $i$ th rule has the form

Rule i: IF $\dot{x}_{i}$ is $A_{1 i}$ and $\dot{y}_{i}$ is $A_{2 i}$ and $x_{i}$ is $A_{3 i}$ and $y_{i}$ is $A_{4 i}$

$$
\text { THEN } \widehat{P}\left(\dot{q}, q \mid \boldsymbol{\theta}_{P}\right)_{i} \text { is } B_{i}, i=1,2, \ldots, M \text {. }
$$

Based on singleton fuzzifier, product inference, and centeraverage defuzzifier, its output can be expressed as

$$
\widehat{P}\left(\dot{q}, q \mid \boldsymbol{\theta}_{P}\right)=\boldsymbol{\theta}_{P}^{T} \boldsymbol{\mu}(\dot{q}, q),
$$

where $\boldsymbol{\mu}(\dot{q}, q)=\left(\boldsymbol{\eta}_{A_{1 i}} \times \boldsymbol{\eta}_{A_{2 i}} \times \boldsymbol{\eta}_{A_{3 i}} \times \boldsymbol{\eta}_{A_{4 i}}\right) /\left(\sum_{j=1}^{M} \boldsymbol{\eta}_{A_{1 j}} \times \boldsymbol{\eta}_{A_{2 j}} \times\right.$ $\left.\boldsymbol{\eta}_{A_{3 j}} \times \boldsymbol{\eta}_{A_{4 j}}\right), \boldsymbol{\eta}_{A_{1 i}}, \boldsymbol{\eta}_{A_{2 i}}, \boldsymbol{\eta}_{A_{3 i}}, \boldsymbol{\eta}_{A_{4 i}}$ are membership function values of the fuzzy variables $\dot{x}, \dot{y}, x, y$ with respect to fuzzy sets $A_{1}, A_{2}, A_{3}, A_{4}$, respectively.

The fuzzy sets of input variables are defined as $\{N, Z, P\}$, where $N$ is negative, $Z$ is zero, and $P$ is positive. Then
TABLE 1: Fuzzy control rules.

\begin{tabular}{llll}
\hline$\dot{x}_{i}$ & $\mathrm{~N}$ & $\mathrm{Z}$ & $\mathrm{P}$ \\
$\dot{y}_{i}$ & $\mathrm{~N}$ & $\mathrm{Z}$ & $\mathrm{P}$ \\
$x_{i}$ & $\mathrm{~N}$ & $\mathrm{Z}$ & $\mathrm{P}$ \\
$y_{i}$ & $\mathrm{~N}$ & $\mathrm{Z}$ & $\mathrm{P}$ \\
\hline
\end{tabular}

N: negative; Z: zero; P: positive.

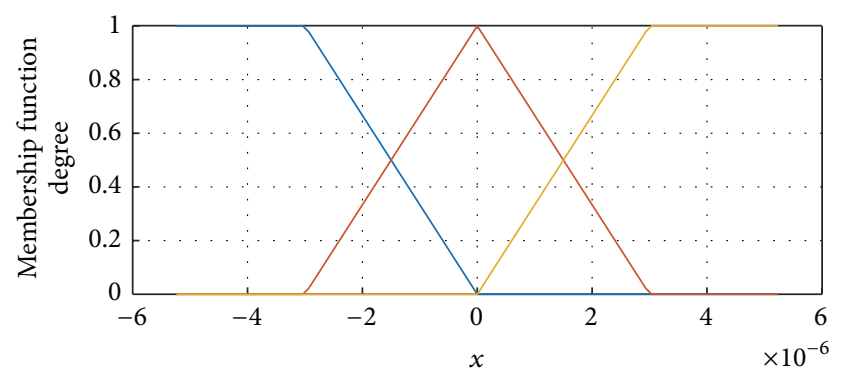

FIGURE 3: The membership functions for $\dot{x}_{i}$.

membership functions of $\dot{x}$ are selected as the following triangular functions:

$$
\begin{aligned}
& \boldsymbol{\eta}_{N}\left(\dot{x}_{i}\right)= \begin{cases}1 & x \leq-3 \\
-\frac{1}{3} x & -3 \leq x \leq 0\end{cases} \\
& \boldsymbol{\eta}_{Z}\left(\dot{x}_{i}\right)= \begin{cases}\frac{1}{3} x+1 & -3 \leq x \leq 0 \\
-\frac{1}{3} x+1 & 0 \leq x \leq 3\end{cases}
\end{aligned}
$$

$$
\boldsymbol{\eta}_{P}\left(\dot{x}_{i}\right)= \begin{cases}1 & x \geq 3 \\ \frac{1}{3} x & 0 \leq x \leq 3\end{cases}
$$

The corresponding membership functions of these fuzzy sets labels are depicted in Figure 3. In addition, the membership functions of $\dot{y}_{i}, x_{i}$, and $y_{i}$ are the same with $\dot{x}_{i}$.

Based on the aforementioned fuzzy sets and membership functions, the fuzzy rules are described in Table 1. Therefore, 81 fuzzy rules are chosen.

The control target for MEMS gyroscope is to maintain the proof mass oscillation at given frequency and amplitude, such as $x_{d}=A_{x} \sin \left(\omega_{x} t\right), y_{d}=A_{y} \sin \left(\omega_{y} t\right)$ in the $x$ and $y$ directions, respectively. So, reference model can be designed as

$$
\ddot{q}_{d}=A_{d} q_{d}
$$

where $q_{d}=\left[\begin{array}{l}x_{d} \\ y_{d}\end{array}\right], A_{d}=\left[\begin{array}{cc}-\omega_{x}^{2} & 0 \\ 0 & -\omega_{y}^{2}\end{array}\right]$.

And the tracking error is defined as

$$
e=q-q_{d}
$$

Nonlinear sliding mode hypersurface is chosen as

$$
s=\dot{e}+\boldsymbol{\alpha} e^{n_{1} / m_{1}}+\boldsymbol{\beta} e^{m_{2} / n_{2}},
$$


where $\boldsymbol{\alpha}>0, \boldsymbol{\beta}>0 ; m_{1}>n_{1}>0, m_{2}>n_{2}>0$; what is more, $m_{1}, n_{1}, m_{2}, n_{2}$ are odd.

Then the reaching law is designed as the following double exponential function:

$$
\dot{s}=-k_{1}|s|^{a} \operatorname{sgn}(s)-k_{2}|s|^{b} \operatorname{sgn}(s)
$$

where $k_{1}>0, k_{2}>0,0<a<1, b>1$.

It should be noted that the converge speed depends on parameters such as $\boldsymbol{\alpha}, \boldsymbol{\beta}, m_{1}, n_{1}, m_{2}, n_{2}$ and $k_{1}, k_{2}, a, b$.

According to (6), equivalent control law is obtained as

$$
\begin{aligned}
u_{\mathrm{eq}} & =C^{-1}\left(\ddot{q}-A \dot{q}-B q-\widehat{P}\left(\dot{q}, q \mid \boldsymbol{\theta}_{P}\right)\right) \\
& =C^{-1}\left[\left(\ddot{q}_{d}+\dot{e}\right)-A \dot{q}-B q-\widehat{P}\left(\dot{q}, q \mid \boldsymbol{\theta}_{P}\right)\right] .
\end{aligned}
$$

And the derivative of sliding surface (11) is

$$
\dot{s}=\ddot{e}+\boldsymbol{\alpha}\left(\frac{n_{1}}{m_{1}}\right) e^{n_{1} / m_{1}-1}+\boldsymbol{\beta}\left(\frac{m_{2}}{n_{2}}\right) e^{m_{2} / n_{2}-1} .
$$

Then substituting (12) into (14),

$$
\begin{aligned}
\ddot{e}= & -k_{1}|s|^{a} \operatorname{sgn}(s)-k_{2}|s|^{b} \operatorname{sgn}(s)-\boldsymbol{\alpha}\left(\frac{n_{1}}{m_{1}}\right) e^{n_{1} / m_{1}-1} \\
& -\boldsymbol{\beta}\left(\frac{m_{2}}{n_{2}}\right) e^{m_{2} / n_{2}-1} .
\end{aligned}
$$

And substituting (15) into (13),

$$
\begin{aligned}
u_{\mathrm{eq}} & =C^{-1}\left[\ddot{q}_{d}-k_{1}|s|^{a} \operatorname{sgn}(s)-k_{2}|s|^{b} \operatorname{sgn}(s)\right. \\
& -\boldsymbol{\alpha}\left(\frac{n_{1}}{m_{1}}\right) e^{n_{1} / m_{1}-1}-\boldsymbol{\beta}\left(\frac{m_{2}}{n_{2}}\right) e^{m_{2} / n_{2}-1}-A \dot{q}-B q \\
& \left.-\widehat{P}\left(\dot{q}, q \mid \boldsymbol{\theta}_{P}\right)\right] .
\end{aligned}
$$

Besides, a robust item is designed to guarantee that the system is asymptotically stable:

$$
u_{s}=-C^{-1} K s \text {. }
$$

Thus, the adaptive fuzzy finite time sliding mode controller is obtained as

$$
u=u_{\mathrm{eq}}+u_{s}
$$

According to (10), we have

$$
\ddot{e}=\ddot{q}-\ddot{q}_{d}=A \dot{q}+B q+C u+P(\dot{q}, q, t)-\ddot{q}_{d} .
$$

Substituting (18) into (19),

$$
\begin{aligned}
\ddot{e} & =A \dot{q}+B q+\left[\ddot{q}_{d}-k_{1}|s|^{a} \operatorname{sgn}(s)-k_{2}|s|^{b} \operatorname{sgn}(s)\right. \\
& \left.-\boldsymbol{\alpha}\left(\frac{n_{1}}{m_{1}}\right) e^{n_{1} / m_{1}-1}-\boldsymbol{\beta}\left(\frac{m_{2}}{n_{2}}\right) e^{m_{2} / n_{2}-1}\right]-A \dot{q} \\
& -B q-\widehat{P}\left(\dot{q}, q \mid \boldsymbol{\theta}_{P}\right)-K s+P(\dot{q}, q, t)-\ddot{q}_{d} \\
& =P(\dot{q}, q, t)-\widehat{P}\left(\dot{q}, q \mid \boldsymbol{\theta}_{P}\right)-K s-k_{1}|s|^{a} \operatorname{sgn}(s) \\
& -k_{2}|s|^{b} \operatorname{sgn}(s)-\boldsymbol{\alpha}\left(\frac{n_{1}}{m_{1}}\right) e^{n_{1} / m_{1}-1}-\boldsymbol{\beta}\left(\frac{m_{2}}{n_{2}}\right) \\
& \cdot e^{m_{2} / n_{2}-1} .
\end{aligned}
$$

Substituting (20) into (14),

$$
\begin{aligned}
\dot{s}= & P(\dot{q}, q, t)-\widehat{P}\left(\dot{q}, q \mid \boldsymbol{\theta}_{P}\right)-K s-k_{1}|s|^{a} \operatorname{sgn}(s) \\
& -k_{2}|s|^{b} \operatorname{sgn}(s) .
\end{aligned}
$$

The optimal parameters are set as

$$
\begin{array}{r}
\boldsymbol{\theta}_{P}^{*}=\arg \min \left[\sup \left|\widehat{P}\left(\dot{q}, q \mid \boldsymbol{\theta}_{P}\right)-P(\dot{q}, q, t)\right|\right] \\
\boldsymbol{\theta}_{P} \in \boldsymbol{\Omega}_{P}, \dot{q}, q \in R^{2 \times 2},
\end{array}
$$

where $\boldsymbol{\Omega}_{P}$ is a set of $\boldsymbol{\theta}_{P}$.

And the minimum approximation errors are defined as

$$
w=P(\dot{q}, q, t)-\widehat{P}\left(\dot{q}, q \mid \boldsymbol{\theta}_{P}\right) .
$$

Substituting (23) into (21), we derive

$$
\begin{aligned}
\dot{s}= & \widehat{P}\left(\dot{q}, q \mid \boldsymbol{\theta}_{P}^{*}\right)-\widehat{P}\left(\dot{q}, q \mid \boldsymbol{\theta}_{P}\right)+w-K s \\
& -k_{1}|s|^{a} \operatorname{sgn}(s)-k_{2}|s|^{b} \operatorname{sgn}(s) .
\end{aligned}
$$

Considering (7), (24) can be expressed as

$$
\begin{aligned}
\dot{s}= & \boldsymbol{\varphi}_{P}^{T} \boldsymbol{\mu}(\dot{q}, q)+w-K s-k_{1}|s|^{a} \operatorname{sgn}(s) \\
& -k_{2}|s|^{b} \operatorname{sgn}(s),
\end{aligned}
$$

where $\boldsymbol{\varphi}_{P}=\boldsymbol{\theta}_{P}^{*}-\boldsymbol{\theta}_{P}$.

So adaptive law can be selected as

$$
\dot{\boldsymbol{\varphi}}_{P}=-r s^{T} \boldsymbol{\mu}(\dot{q}, q) .
$$

Namely,

$$
\begin{aligned}
& \dot{\boldsymbol{\theta}}_{P x}=r s(1) \boldsymbol{\mu}_{x}(\dot{q}, q), \\
& \dot{\boldsymbol{\theta}}_{P y}=r s(2) \boldsymbol{\mu}_{y}(\dot{q}, q),
\end{aligned}
$$

where $\dot{\boldsymbol{\varphi}}_{P}=-\dot{\boldsymbol{\theta}}_{P}$.

Lyapunov function is defined as

$$
V=\frac{1}{2}\left(s^{T} s+\frac{1}{r} \boldsymbol{\varphi}_{P}^{T} \boldsymbol{\varphi}_{P}\right) .
$$


Differentiate $V$ with respect to time yields, and substitute (26) as

$$
\begin{aligned}
\dot{V}= & s^{T} w-s^{T} K s-k_{1} s^{T}|s|^{a} \operatorname{sgn}(s) \\
& -k_{2} s^{T}|s|^{b} \operatorname{sgn}(s) .
\end{aligned}
$$

Owing to the fuzzy approximation theory, adaptive fuzzy system can approximate nonlinear system closely. Therefore, $\dot{V} \leq 0$; namely, the system is asymptotically stable.

\section{Simulation Study}

In this section, numerical simulations are investigated to track the position and speed trajectories of MEMS gyroscope, compensate parametric uncertainties and external disturbances, and verify the superiority of the proposed approach compared with conventional adaptive fuzzy sliding mode control strategy using linear sliding mode surface. Those two methods are defined as follows.

Method 1. Define the adaptive fuzzy sliding mode control proposed in this paper as Method 1, whose sliding mode surface is shown in (11), and the reaching law is expressed in (12).

Method 2. Define the conventional adaptive fuzzy sliding mode control as Method 2, whose sliding mode surface is $=\dot{e}+\beta e$, and the reaching law is $\dot{s}=0$.

Parameters of the MEMS gyroscope are as follows:

$$
\begin{aligned}
m & =0.57 \times 10^{-8} \mathrm{~kg}, \\
d_{x x} & =0.429 \times 10^{-6} \mathrm{Ns} / \mathrm{m}, \\
d_{y y} & =0.0429 \times 10^{-6} \mathrm{Ns} / \mathrm{m}, \\
d_{x y} & =0.0429 \times 10^{-6} \mathrm{Ns} / \mathrm{m}, \\
k_{x x} & =80.98 \mathrm{~N} / \mathrm{m}, \\
k_{y y} & =71.62 \mathrm{~N} / \mathrm{m}, \\
k_{x y} & =5 \mathrm{~N} / \mathrm{m}, \\
\Omega_{z} & =5.0 \mathrm{rad} / \mathrm{s} .
\end{aligned}
$$

Since the position of proof mass ranges within the scope of submillimeter and the natural frequency is generally in the range of kilohertz, assume that reference length is $q_{o}=$ $10 \times 10^{-6} \mathrm{~m}$, reference frequency is $\omega_{o}=1 \mathrm{kHz}$, and the reference trajectories are $x_{d}=\sin (6.71 t), y_{d}=1.2 \sin (5.11 t)$, respectively.

Then set other simulation parameters as

$$
\begin{aligned}
& A=\left[\begin{array}{cc}
-0.075 & 0.0025 \\
-0.0175 & -0.0075
\end{array}\right], \\
& B=\left[\begin{array}{cc}
-14207 & -877 \\
-877 & -12564
\end{array}\right],
\end{aligned}
$$
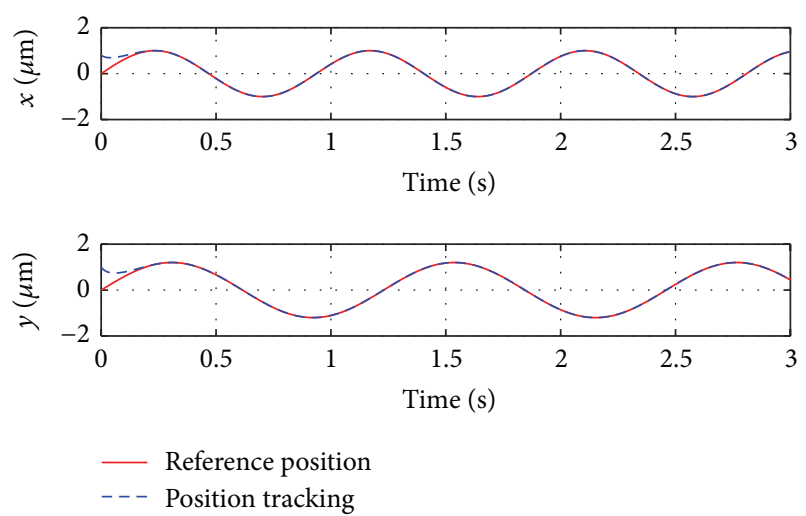

FIgure 4: Position tracking of Method 1.

$$
\begin{aligned}
& C=\left[\begin{array}{ll}
1 & 0 \\
0 & 1
\end{array}\right], \\
& K=\left[\begin{array}{cc}
1000 & 0 \\
0 & 1000
\end{array}\right] \text {, } \\
& \boldsymbol{\alpha}=\left[\begin{array}{cc}
10 & 0 \\
0 & 10
\end{array}\right] \text {, } \\
& \boldsymbol{\beta}=\left[\begin{array}{cc}
10 & 0 \\
0 & 10
\end{array}\right] \text {, } \\
& m_{1}=3 \text {, } \\
& n_{1}=2 \text {, } \\
& m_{2}=3 \text {, } \\
& n_{2}=1 \text {, } \\
& P(t)=\left[\begin{array}{c}
3.2 \times 10^{-6} \\
5 \times 10^{-6}+5 \times 10^{-6} \sin (5.11(t+0.3))
\end{array}\right], \\
& r=0.01 \text {, } \\
& a=0.5 \text {, } \\
& b=10, \\
& k_{1}=1000 \text {, } \\
& k_{2}=1000 \text {. }
\end{aligned}
$$

And select the initial state values of the system as $\left[\begin{array}{llll}0.8 & 0 & 1 & 0\end{array}\right]^{T}$.

Then the position and speed trajectories of Method 1 are shown in Figures 4 and 5 and those of Method 2 are depicted in Figures 6 and 7.

The position tracking error and speed tracking error of Methods 1 and 2 are shown in Figures 8-11, respectively.

Through the tracking simulation of MEMS gyroscope, the proposed approach is with satisfying performance; in 

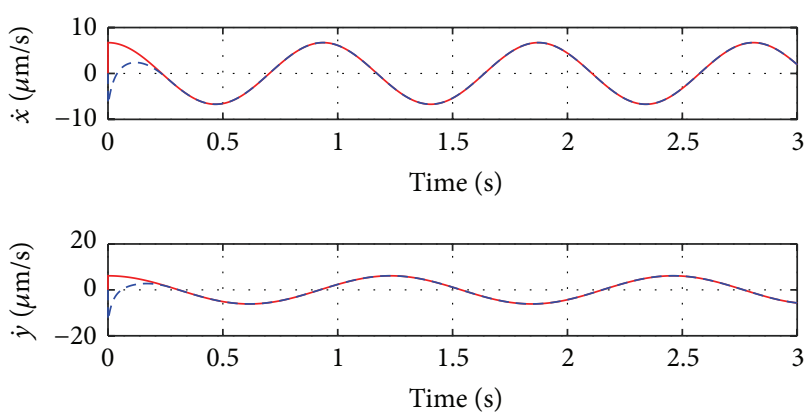

- Reference speed

- - Speed tracking

FIGURE 5: Speed tracking of Method 1.
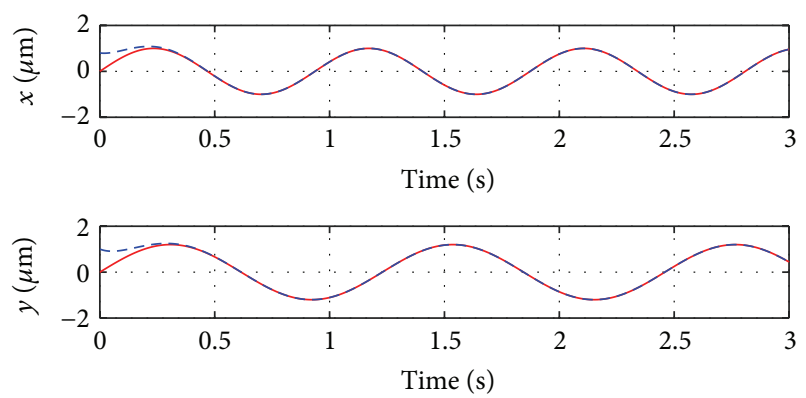

Reference position

- - Position tracking

Figure 6: Position tracking of Method 2.
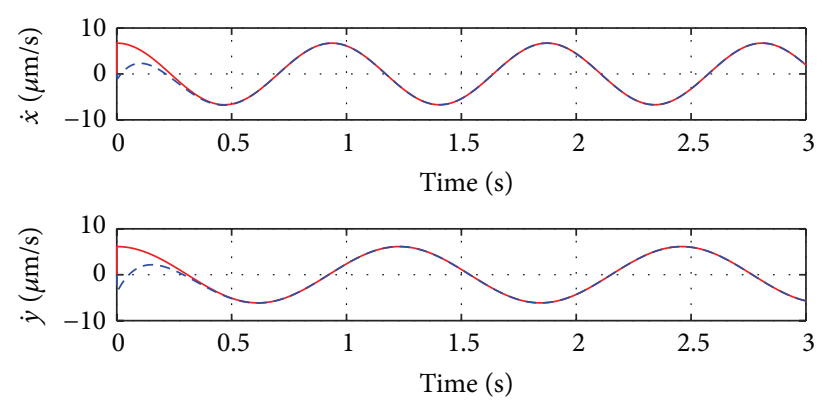

- Reference speed

- - Speed tracking

FIGURE 7: Speed tracking of Method 2.

addition, in comparison to Method 2, the convergence time is shortened to $0.3^{\prime \prime}$ from $0.6^{\prime \prime}$.

\section{Conclusion and Future Work}

An adaptive fuzzy finite time sliding mode control strategy using nonlinear sliding mode hypersurface and double exponential reaching law is proposed to compensate parametric uncertainties and external disturbance of MEMS gyroscope in this paper. Based on Lyapunov methods, the stability of system can be guaranteed. Simulations verify that, compared

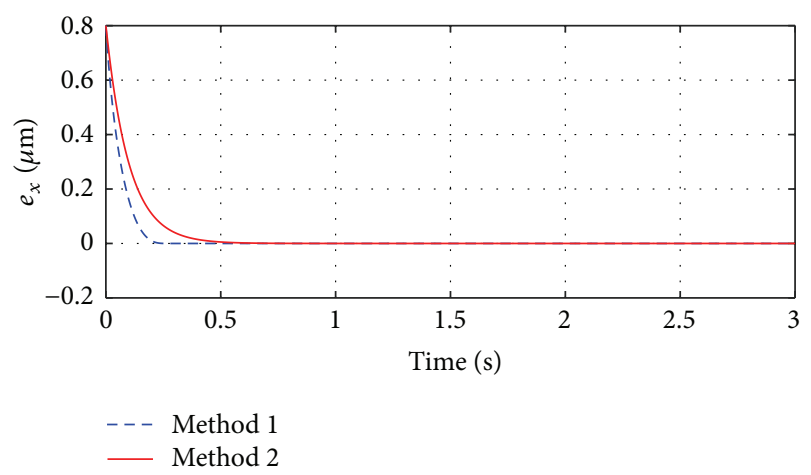

FIGURE 8: Position tracking error of gyroscope $x$.

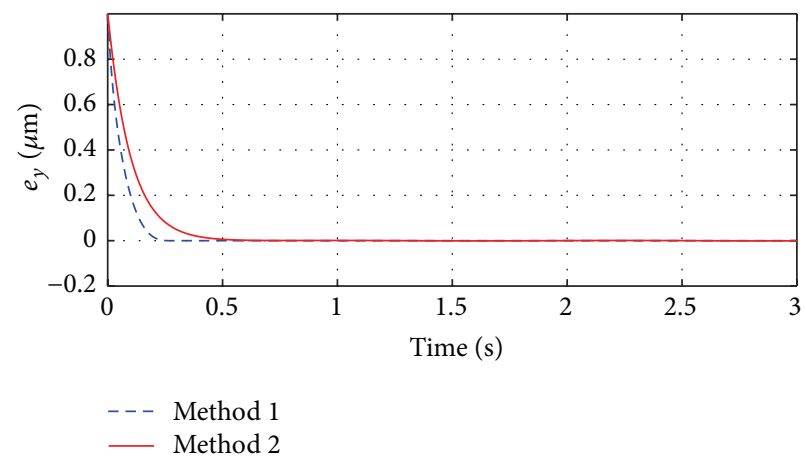

FIGURE 9: Position tracking error of gyroscope $y$.

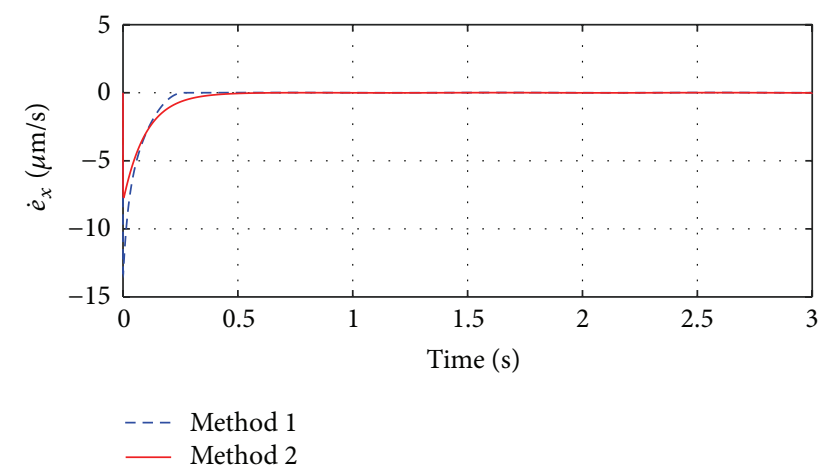

FIGURE 10: Speed tracking error of gyroscope $x$.

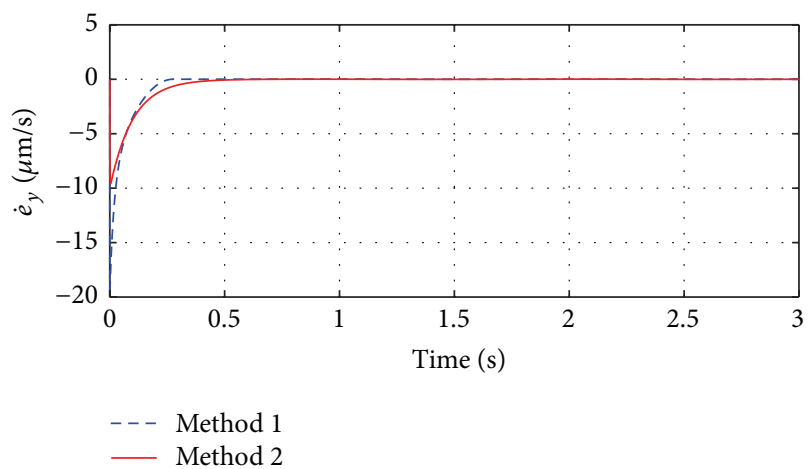

FIGURE 11: Speed tracking error of gyroscope $y$. 
with conventional adaptive fuzzy linear sliding mode control strategy, the convergence time of finite time convergent control strategy proposed in this paper is shortened to $0.3^{\prime \prime}$ from $0.6^{\prime \prime}$; namely, convergence has been significantly improved. For future work, the novel adaptive online constructing fuzzy algorithm [24] can be employed for more efficient learning while disturbance observer based design $[25,26]$ can be considered to improve system performance.

\section{Competing Interests}

The authors declare that they have no competing interests.

\section{Acknowledgments}

This work was supported by National Natural Science Foundation of China (61304098, 60204005, and 60974109), Aeronautical Science Foundation of China (2015ZA53003), Natural Science Basic Research Plan in Shaanxi Province (2014JQ8326, 2015JM6272, and 2016KJXX-86), Fundamental Research Funds for the Central Universities (3102015AX001, 3102015BJ(II)CG017), Fundamental Research Funds of Shenzhen Science and Technology Project (JCYJ20160229172341417), and International Science and Technology Cooperation Program of China under Grant no. 2014DFA11580.

\section{References}

[1] M. Chen and S. S. Ge, "Adaptive neural output feedback control of uncertain nonlinear systems with unknown hysteresis using disturbance observer," IEEE Transactions on Industrial Electronics, vol. 62, no. 12, pp. 7706-7716, 2015.

[2] B. Xu, C. Yang, and Y. Pan, "Global neural dynamic surface tracking control of strict-feedback systems with application to hypersonic flight vehicle," IEEE Transactions on Neural Networks and Learning Systems, vol. 26, no. 10, pp. 2563-2575, 2015.

[3] B. Xu, Y. Fan, and S. Zhang, "Minimal-learning-parameter technique based adaptive neural control of hypersonic flight dynamics without back-stepping," Neurocomputing, vol. 164, pp. 201-209, 2015.

[4] Q. Yang, S. Jagannathan, and Y. Sun, "Robust integral of neural network and error sign control of MIMO nonlinear systems," IEEE Transactions on Neural Networks and Learning Systems, vol. 26, no. 12, pp. 3278-3286, 2015.

[5] B. Xu, Q. Zhang, and Y. Pan, "Neural network based dynamic surface control of hypersonic flight dynamics using small-gain theorem," Neurocomputing, vol. 173, pp. 690-699, 2016.

[6] Y.-J. Liu and S. Tong, "Adaptive NN tracking control of uncertain nonlinear discrete-time systems with nonaffine dead-zone input," IEEE Transactions on Cybernetics, vol. 45, no. 3, pp. 497$505,2015$.

[7] W. He, Y. Chen, and Z. Yin, "Adaptive neural network control of an uncertain robot with full-state constraints," IEEE Transactions on Cybernetics, vol. 46, no. 3, pp. 620-629, 2016.

[8] S. Park and R. Horowitz, "New adaptive mode of operation for MEMS gyroscopes," ASME Journal of Dynamic Systems, Measurement and Control, vol. 126, no. 4, pp. 800-810, 2004.
[9] C. Batur, T. Sreeramreddy, and Q. Khasawneh, "Sliding mode control of a simulated MEMS gyroscope," ISA Transactions, vol. 45, no. 1, pp. 99-108, 2006.

[10] A. Ebrahimi, "Regulated model-based and non-model-based sliding mode control of a MEMS vibratory gyroscope," Journal of Mechanical Science and Technology, vol. 28, no. 6, pp. 23432349, 2014.

[11] J. Fei and C. Batur, "A novel adaptive sliding mode control with application to MEMS gyroscope," ISA Transactions, vol. 48, no. 1, pp. 73-78, 2009.

[12] Z. A. K. Michail, "Terminal attractors in neural networks," Neural Networks, vol. 2, no. 2, pp. 259-274, 1989.

[13] X. Yu and M. Zhihong, "Fast terminal sliding-mode control design for nonlinear dynamical systems," IEEE Transactions on Circuits and Systems I: Fundamental Theory and Applications, vol. 49, no. 2, pp. 261-264, 2002.

[14] X. Yu and Z. Man, "Terminal sliding mode control of MIMO systems," IEEE Trans on Circuits Systems I, vol. 44, no. 11, pp. 1065-1070, 1997.

[15] A. Ghanbari and M. R. Moghanni-Bavil-Olyaei, "Adaptive fuzzy terminal sliding-mode control of MEMS z-axis gyroscope with extended Kalman filter observer," Systems Science \& Control Engineering, vol. 2, pp. 183-191, 2014.

[16] W. F. Yan, S. X. Hou, Y. M. Fang, and J. Fei, "Robust adaptive nonsingular terminal sliding mode control of MEMS gyroscope using fuzzy-neural-network compensator," International Journal of Machine Learning and Cybernetics, 2016.

[17] A. Bartoszewicz, "A new reaching law for sliding mode control of continuous time systems with constraints," Transactions of the Institute of Measurement and Control, vol. 37, no. 4, pp. 515-521, 2015.

[18] W. Gao and J. C. Hung, "Variable structure control of nonlinear systems: a new approach," IEEE Transactions on Industrial Electronics, vol. 40, no. 1, pp. 45-55, 1993.

[19] C. J. Fallaha, M. Saad, H. Y. Kanaan, and K. Al-Haddad, "Sliding-mode robot control with exponential reaching law," IEEE Transactions on Industrial Electronics, vol. 58, no. 2, pp. 600-610, 2011.

[20] H. Mei and Y. Wang, "Fast convergent sliding mode variable structure control of robot," Information and Control, vol. 38, no. 5, pp. 552-557, 2009.

[21] L.-X. Wang and J. M. Mendel, "Fuzzy basis functions, universal approximation, and orthogonal least-squares learning," IEEE Transactions on Neural Networks, vol. 3, no. 5, pp. 807-814, 1992.

[22] X. Zeng and M. G. Singh, "Singh approximation theory of fuzzy systems-MIMO case," IEEE Transactions on Fuzzy Systems, vol. 3, no. 2, pp. 219-235, 1995.

[23] J. Fei and S. Wang, "Robust adaptive sliding mode control of MEMS gyroscope using T-S fuzzy model," Nonlinear Dynamics, vol. 77, no. 1-2, pp. 361-371, 2014.

[24] N. Wang and M. J. Er, "Direct adaptive fuzzy tracking control of marine vehicles with fully unknown parametric dynamics and uncertainties," IEEE Transactions on Control Systems Technology, vol. 24, no. 5, pp. 1845-1852, 2016.

[25] B. Xu, F. Sun, Y. Pan, and B. Chen, "Disturbance observer based composite learning fuzzy control of nonlinear systems with unknown dead zone," IEEE Transactions on Systems, Man, and Cybernetics: Systems, 2016.

[26] B. Xu, "Disturbance observer-based dynamic surface control of transport aircraft with continuous heavy cargo airdrop," IEEE Transactions on Systems, Man, and Cybernetics: Systems, 2016. 


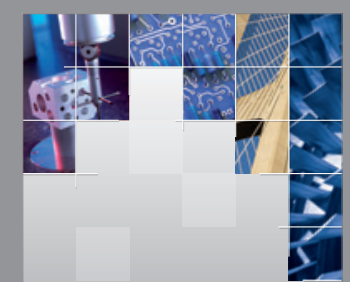

\section{Enfincering}
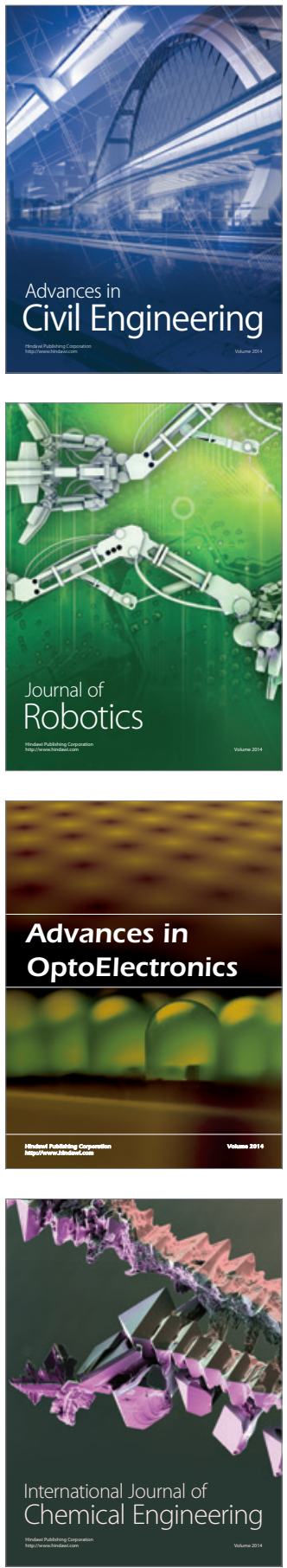

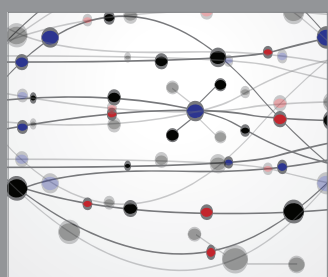

The Scientific World Journal

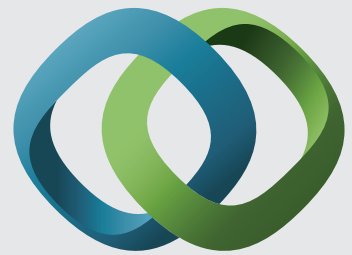

\section{Hindawi}

Submit your manuscripts at

http://www.hindawi.com
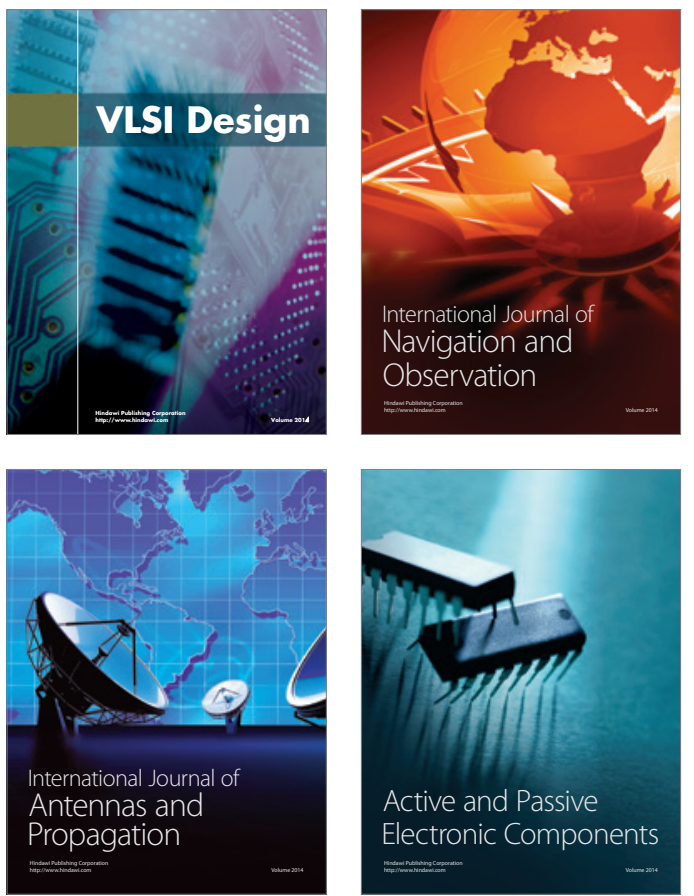
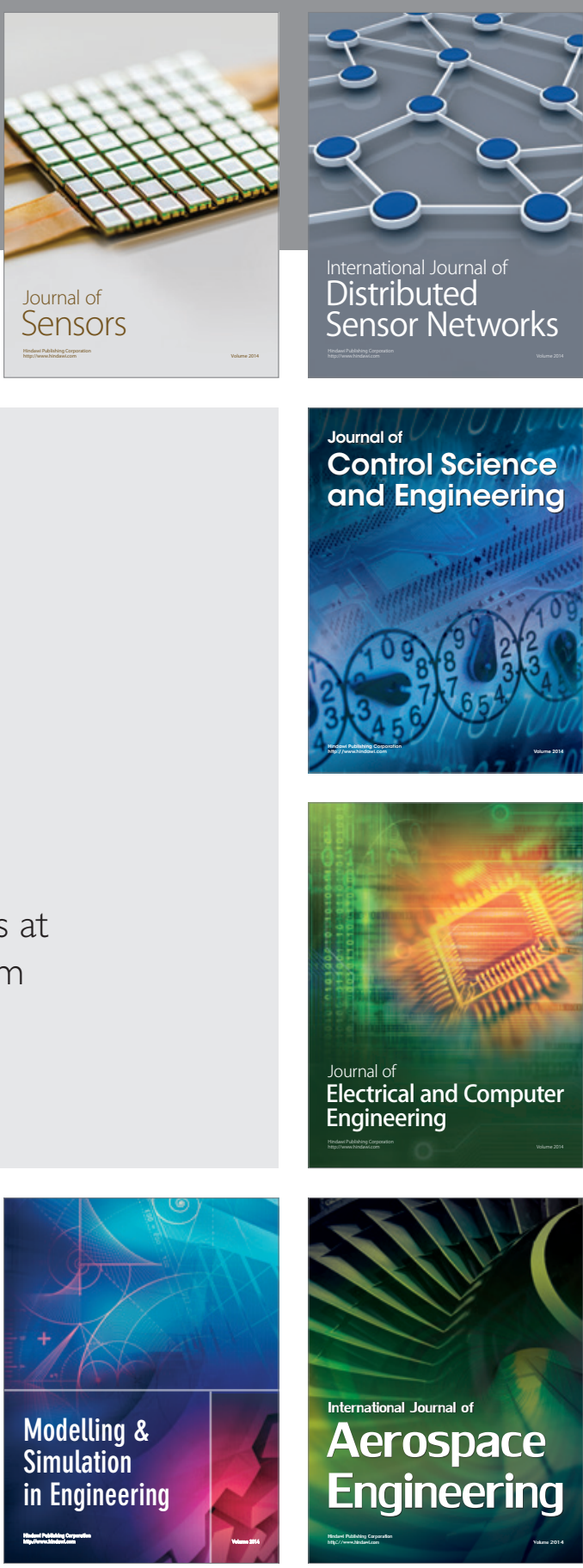

International Journal of

Distributed

Sensor Networks

Journal of

Control Science

and Engineering
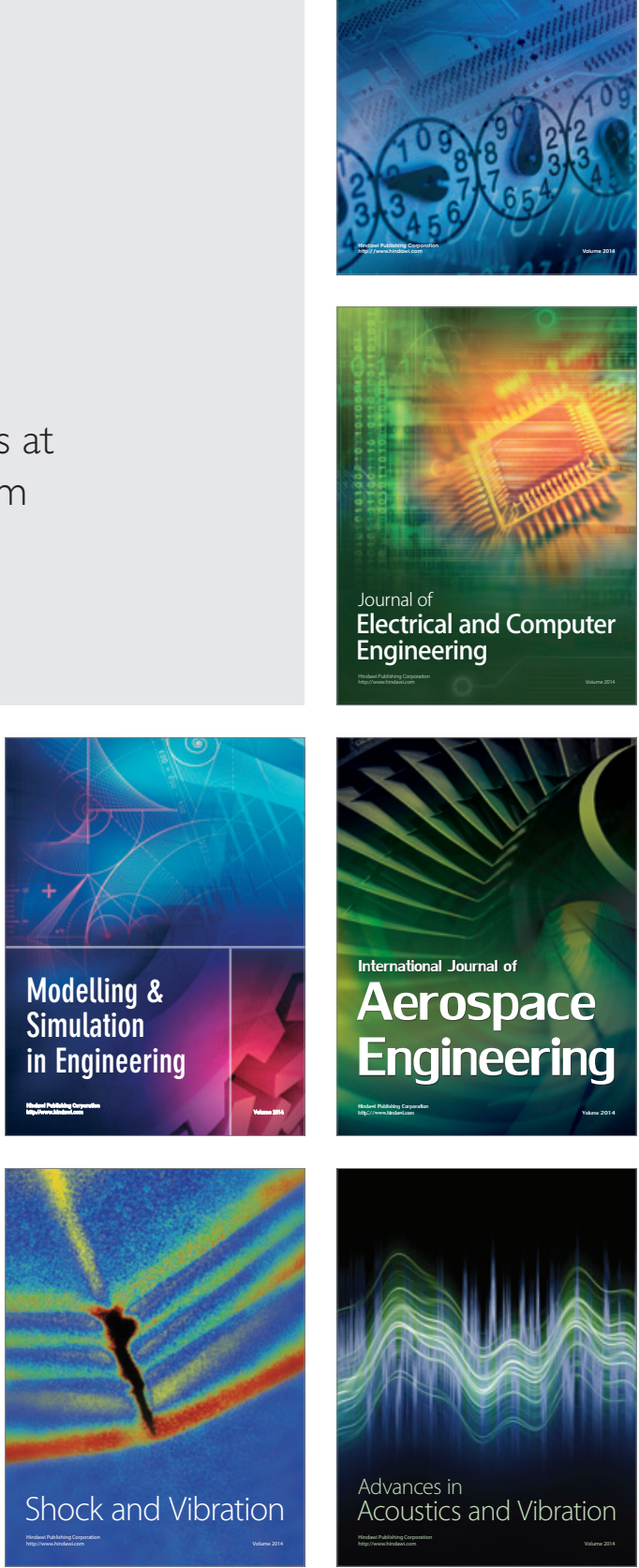